\title{
Millimeter-Wave and Sub-THz Modulated Metasurface Antennas
}

\author{
$\underline{\text { D. González-Ovejero }}^{1}$, X. Morvan ${ }^{1}$, L. Le Coq, and O. de $\operatorname{Sagazan}^{1}$ \\ ${ }^{1}$ Univ. Rennes, CNRS, IETR (Institut d'Électronique et de Télécommunications de Rennes) - UMR 6164, \\ F-35000, Rennes, France. \\ david.gonzalez-ovejero@univ-rennes1.fr
}

\begin{abstract}
Modulated metasurfaces (MTSs) have sprung up in the last decade as an attractive solution for wave guidance and radiation. More precisely, modulated MTS antennas stand out for providing an unprecedented control of the aperture fields with low-profile and lightweight structures. In this class of antennas, a surface-wave (SW) is gradually radiated, owing to its interaction with a modulated impedance boundary condition (IBC). This IBC is typically implemented in the microwave regime by several thousands of sub-wavelength patches printed on a grounded slab. However, dielectric losses may hinder the use of standard printed circuit board (PCB) technology at higher frequencies. In this paper, we will address this issue by introducing a new class of metal-only modulated MTS, which can be easily fabricated by additive manufacturing or micro-machining for millimeter-wave and sub-THz applications.
\end{abstract}

\section{INTRODUCTION}

Modulated metasurfaces (MTSs) can be efficiently used to guide the propagation of surface-wave (SW) wavefronts [1] or to gradually radiate the power carried by a SW [2]. Formally, the MTS aperture can be represent by a space-varying anisotropic impedance boundary condition (IBC). The IBC modulation in MTS antennas is typically periodic and, by properly choosing the period of this modulation, the -1 mode in the Floquet expansion can enter the visible region and provide the desired radiation effect. Besides polarized and shaped beams [2], this class of antenna can provide multiple-beams [3] and broadband operation [4].

In the microwave regime, modulated MTS antennas typically consist of several thousands of sub-wavelength patches printed on a grounded slab and fabricated by standard PCB technology. However, dielectric losses may significantly reduce their radiation efficiency for millimeter-wave and sub-THz applications. Although one could use either polymers (e.g. benzocyclobutene or cyclic olefin copolymer) or high-resistivity silicon as high-performance substrates, metal-only solutions constitute an extremely promising alternative. Indeed, metal-only designs will be less susceptible to thermal variation and will not suffer for dielectric property change due to a high level of radiation [5]. Such properties are advantageous, for instance, to withstand harsh environments in space exploration.

This paper presents a class of modulated MTS antennas that can be fabricated entirely in metal, and it is organized as follows. First, we will succinctly describe the design process and present the unit-cell that enables the metallic implementation of the IBC. Then, two different fabrication techniques are discussed, along with their advantages and limitations. Finally, conclusions are drawn.

\section{Metal-only modulated MTS antennas}

In the remainder of this paper we will consider, without loss of generality, circular apertures and a cylindrical reference system with unit vectors $(\hat{\boldsymbol{\rho}}, \hat{\boldsymbol{\phi}})$. In a nutshell, the design problem consists in finding an impenetrable IBC $\underline{\underline{\mathbf{X}}}(\boldsymbol{\rho})$ that provides the desired aperture fields $\mathbf{E}_{A}$. This IBC relates the tangential fields on the MTS aperture as

$$
\left.\mathbf{E}_{t}\right|_{z=0^{+}}=\mathrm{j} \underline{\underline{\mathbf{X}}}(\boldsymbol{\rho}) \cdot \hat{\mathrm{z}} \times\left.\mathbf{H}_{t}\right|_{z=0^{+}}
$$



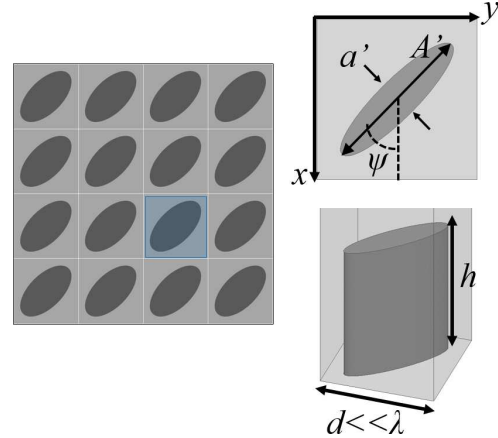

(a)

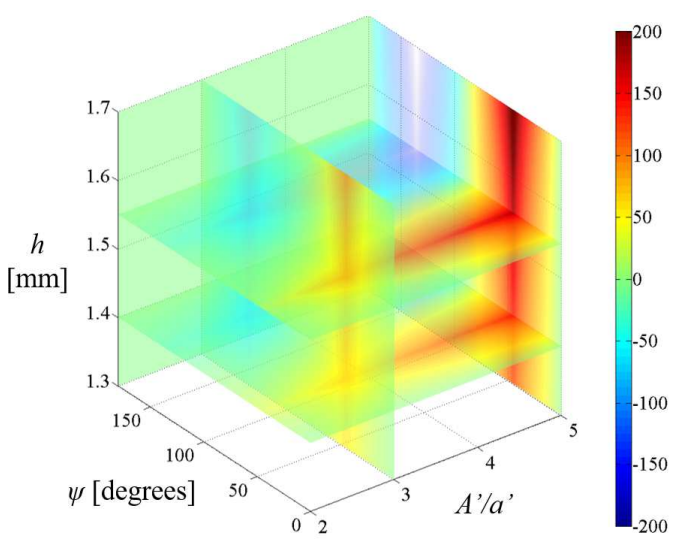

(b)

Fig. 1: (a) Description of the unit-cell geometry and local periodicity assumption. (b) Example of database relating the geometry of the unit-cell with the corresponding reactance tensor.

where $\left.\mathbf{E}_{t}\right|_{z=0^{+}}$and $\left.\mathbf{H}_{t}\right|_{z=0^{+}}$are the total tangential electric and magnetic fields, respectively. Following the procedure described in [6], one can calculate $\underline{\underline{\mathbf{X}}}(\boldsymbol{\rho})$ as

$$
\underline{\underline{\mathbf{X}}}(\boldsymbol{\rho}) \cdot\left\{\begin{array}{l}
\hat{\boldsymbol{\rho}} \\
\hat{\boldsymbol{\phi}}
\end{array}\right\}=\left[\mathrm{X}_{0}\left\{\begin{array}{c}
\hat{\boldsymbol{\rho}} \\
\hat{\boldsymbol{\phi}}
\end{array}\right\} \pm 2 \operatorname{Im}\left(\frac{\mathbf{E}_{A}}{I_{T M} \mathrm{H}_{1}^{(2)}\left(\beta_{s w} \rho\right)}\right)\right] \mathrm{U}_{A}
$$

where $I_{T M}$ is the SW complex excitation coefficient, $\mathrm{H}_{1}^{(2)}(\cdot)$ is the Hankel function of the second kind and first order and $\beta_{s w}$ is the SW wave-number, related to the $\rho$ component of $\underline{\mathbf{X}}$ as $\beta_{s w}=k \sqrt{1+\left(X_{0} / \zeta\right)^{2}}$.

Once the value of $\underline{\mathbf{X}}(\boldsymbol{\rho})$ on the aperture is known, one has to find metallic elements that reproduce the local behavior of the IBC tensor. Here, we explore the use of cylinders with elliptical cross-section (see Fig.1a), as in $[7,6,8]$. To obtain the antenna layout, we first build a database that links the geometry of the cylinders with a reactance tensor. Fig. $1 \mathrm{~b}$ shows the variation of $X_{\rho \rho}$ with the different geometrical features. Since the variation between one element and its neighbors is small (see Fig.1a), we can assume that the structure is locally periodic and use a periodic solver to build our database. Next, $\underline{\underline{\mathbf{X}}}(\boldsymbol{\rho})$ is sampled on a Cartesian lattice, with unit-cell size equal to $d$. Finally, each sample is implemented using a metallic pillar with the geometry retrieved form the database.

The peak gain and relative bandwidth (defined as $\Delta f / f_{0}$, with $\Delta f$ being the total bandwidth and $f_{0}$ the central frequency) of this antennas are closely related. Practical values of $\Delta f / f_{0}$ may vary between $3 \%$ and $9 \%$ when the antenna gain goes from 40 to $28.5 \mathrm{~dB}$. Nonetheless, one can trade gain for larger bandwidths as explained in [4]. Another crucial aspect consists in choosing the most appropriate fabrication technique at each frequency band. The fabrication technique may impose some restrictions in the design phase, for instance, the maximum ratio between cylinder height and unit-cell side. In general, the technological choice is mostly dictated by the dimensions of the MTS unit-cells.

\section{A. Additive manufacturing}

In the millimeter-wave range, we have demonstrated that metal additive manufacturing (AM) can be used to realize structures at Ka band [6]. Fig.2a shows a MTS antenna where the elliptical cylinders have been grown by laser beam melting (LBM) on an aluminum base-plate using AlSi10Mg powder. The feeding circuit (not shown here) was machined on the other side of the base-plate.

\section{B. Micro-machining}

At higher frequencies, the maximum accuracy and surface roughness provided by LBM and similar methods lead to strong limitations in the antenna performance. However, it is possible to use micro-machining as high precision fabrication technique. In this regard, one of the most popular approaches is to use plasma etching, such as deep reactive ion etching (DRIE). This way, one can manufacture the MTS texture with an excellent accuracy 


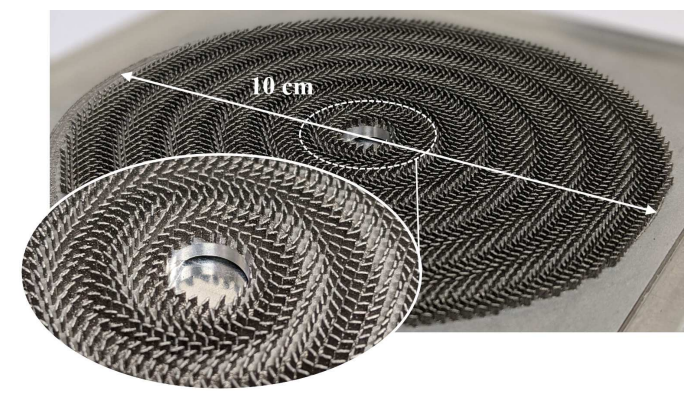

(a)

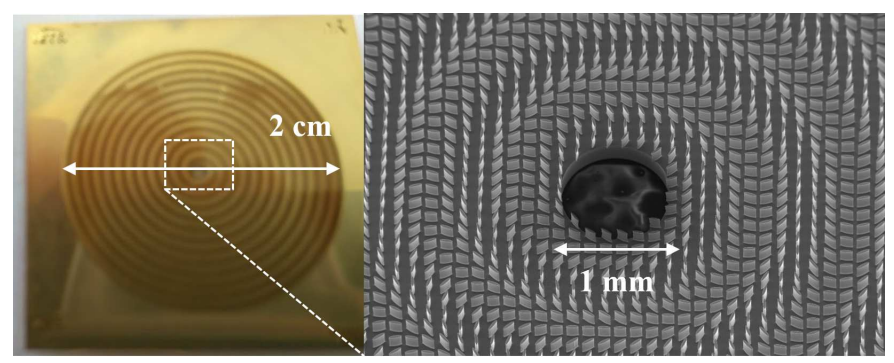

(b)

Fig. 2: (a) Ka-band modulated MTS antenna fabricated by metal additive manufacturing. (b) Modulated MTS antenna operating at $300 \mathrm{GHz}$ and fabricated by DRIE.

by etching a Silicon wafer that is afterward metalized by sputtering [7, 8]. The main drawback of DRIE is that the maximum etching depth cannot be larger than a few hundreds of microns, so it is difficult to apply it at lower frequencies. Fig. $2 \mathrm{~b}$ shows one of our $300 \mathrm{GHz}$ prototypes fabricated by DRIE.

\section{CONCLUSION}

This paper has discussed a metal-only modulated MTS antenna along with two possible fabrication techniques, one for millimeter-waves and the other one in the sub- $\mathrm{THz}$ range. The proposed structure allows one to overcome some of the limitations imposed by the use of dielectric substrates at high frequencies and/or in some applications. At the conference, we will present new designs and further discuss important aspects like the tapering of the aperture fields to increase the aperture efficiency or the use of new modulations to enlarge the bandwidth.

\section{ACKNOWLEDGEMENT}

This publication has been supported by the European Union through the European Regional Development Fund (ERDF), and by the French Region of Brittany, Ministry of Higher Education and Research, Rennes Métropole and Conseil Départemental 35, through the CPER Project STIC \& Ondes. The authors are also grateful to the platform $\mathrm{M}^{2} \mathrm{ARS}$, Université de Rennes 1, and to the platform NanoRennes. The work of D. González-Ovejero was supported by Région Bretagne through the Stratégie d'Attractivité Durable (SAD) volet 2 Programme under Contract SAD17012 and by CNRS-INSIS through the program PEPS Ingénierie Inspirée par la Nature.

\section{REFERENCES}

[1] E. Martini, M. J. Mencagli, D. González-Ovejero, and S. Maci, "Flat optics for surface waves," IEEE Trans. Antennas Propag., vol. 64, no. 1, pp. 155-166, Jan 2016.

[2] M. Faenzi et al., "Metasurface antennas: New models, applications and realizations," Sci. Rep., vol. 9, p. 10178, July 2019.

[3] D. González-Ovejero, G. Minatti, G. Chattopadhyay, and S. Maci, "Multibeam by metasurface antennas," IEEE Trans. Antennas Propag., vol. 65, no. 6, pp. 2923-2930, Jun. 2017.

[4] M. Faenzi, D. González-Ovejero, and S. Maci, "Wideband active region metasurface antennas," IEEE Trans. Antennas Propag., vol. 68, no. 3, pp. 1261-1272, 2020.

[5] N. Chahat, B. Cook, H. Lim, and P. Estabrook, "All-metal dual frequency RHCP high gain antenna for a potential europa lander," IEEE Trans. Antennas Propag., vol. 66, no. 12, pp. 6791-6798, Dec. 2018.

[6] D. González-Ovejero, N. Chahat, R. Sauleau, G. Chattopadhyay, S. Maci, and M. Ettorre, "Additive manufactured metalonly modulated metasurface antennas," IEEE Trans. Antennas Propag., vol. 66, no. 11, pp. 6106-6114, Nov 2018.

[7] D. González-Ovejero, T. J. Reck, C. D. Jung-Kubiak, M. Alonso-DelPino, and G. Chattopadhyay, "A class of silicon micromachined metasurface for the design of high-gain terahertz antennas," in Proc. IEEE Antennas Propag. Soc. Int. Symp., Fajardo, Puerto Rico, Jun. 26 - Jul. 1 2016, pp. 1191-1192.

[8] D. González-Ovejero et al., "Design, fabrication and testing of a modulated metasurface antenna at $300 \mathrm{GHz}$," in Proc. 11th Eur. Conf. Antennas Propag. (EUCAP), Paris, France, Mar. 19-24 2017, pp. 3416-3418. 